\title{
La réécriture tragique d'Héraclius de Corneille pour la France napoléonienne
}

\author{
Clare Siviter \\ Université de Warwick
}

Il existe un rapport étroit entre la tragédie et la réécriture depuis plusieurs siècles, et en France, les tragédies dites «classiques»sont les hypertextes d'hypotextes anciens (voir Genette, p. 13): les dramaturges reprennent des histoires préexistantes, telles que l'Énéide, les transforment et créent une nouvelle tragédie qui répond aux attentes et aux besoins de la société contemporaine, comme ce fut le cas pour l'Andromaque (1667) de Racine. Ensuite, la réécriture continue d'évoluer au fil du temps, modifiée par le dramaturge qui corrige lui-même ses œuvres - tel Pierre Corneille (voir Couton, p. 1466-1471) ou Voltaire (voir Chaouche, p. 133-137) - ou par une autre main, 
comme l'ont confirmé les travaux récents d'Antionio Sergi, d'Eric Eigenmann, de Pierre Frantz, de Sophie Marchand, de Stéphan Zekian et de Sabine Chaouche, lesquels se concentrent sur les transformations qui ont eu lieu pendant les dix-huitième et dix-neuvième siècles.

D’après André Lefevere, toute réécriture est soumise à son contexte idéologique et se fait en relation avec la situation sociale et politique (1992, p. 7-16). Cette affirmation est surtout évidente pour la tragédie «classique» à l'époque révolutionnaire, pendant laquelle les références monarchiques ont été supprimées, le tutoiement entre les personnages introduit et le texte original coupé afin de le rendre plus tragique. Ces modifications sont parfois brutales et, faites à la demande du gouvernement ou même par des individus au sein $\mathrm{du}$ gouvernement, confirment leur motivation politique, telle que dans La Mort de César (1735) de Voltaire en 1793 et 1794, modifiée par le ministre de justice Louis Gohier. En effet cette tragédie réécrite se termine sur un tableau où les hommes sont agenouillés autour d'une statue de la Liberté et acclament: «VIVE LA LIBERTÉ! VIVE LA RÉPBLIQUE! » (Voltaire, 17931794, III, 9, p. 37).

L'analyse de la réécriture d'Héraclius (1647) de Corneille pour les représentations de l'époque napoléonienne montre les enjeux de la réécriture tragique pour la société postrévolutionnaire et pour le régime napoléonien. Cette tragédie, créée lors de l'année théâtrale 1646-1647 par Corneille et imprimée en 1647, est reprise le 7 août 1806 devant Napoléon à Saint-Cloud et le 24 janvier 1807 à Paris à la ComédieFrançaise. Il est fascinant que Napoléon, Empereur des Français au sommet de son pouvoir, accorde son patronage à Héraclius, 
une tragédie qui reprend les thèmes qui ont marqué le théâtre révolutionnaire, tels que les fausses identités et les changements de pouvoir. Le sujet est d'ailleurs très complexe : Héraclius est le fils de Maurice, l'ancien Empereur d'Orient, dont le pouvoir a été usurpé par Phocas, désormais Empereur. Tous les fils de Maurice ont été assassinés mais Héraclius a échappé à son destin : il a été sauvé par Léontine, sa gouvernante, qui, en échange, a sacrifié son propre fils. Ensuite, celle-ci accomplit un deuxième échange entre ce prince et le fils de Phocas, appelé Martian. Ce dernier est élevé en tant que Léonce, fils de Léontine, et Héraclius se croit Martian, fils de Phocas. Phocas souhaite que Martian se marie avec Pulchérie, la fille de Maurice, pour renforcer son pouvoir, mais le projet de ce mariage incestueux qui, inconsciemment, révolte Pulchérie et Martian (Héraclius) pousse Léontine à révéler la naissance d'Héraclius. Pourtant, les vraies identités ne sont qu'à moitié révélées: Léonce (Martian) se croit toujours Héraclius. Phocas, troublé, ne sait ce qu'il faut faire ni à qui il peut faire confiance. Face au peuple, il fait patrouiller ses gardes dans les rues, laissant le palais vulnérable, ce qui aboutit à son meurtre par la main du noble Exupère, qui déteste Phocas. Enfin, grâce au billet de l'ancienne impératrice, mère de Pulchérie et d'Héraclius, Léontine peut enfin révéler qui est de quel sang: Héraclius peut régner tandis que Pulchérie et Martian peuvent s'aimer.

Loin des réécritures révolutionnaires, les autorités de l'époque napoléonienne souhaitent adopter une approche plus subtile à un moment où les esprits se calment, où les canons se refondent et où la nation française se reconstruit. Néanmoins, la réécriture d'Héraclius issue de la Police est, avec treize pages, l'une des plus longues de l'époque. Elle a également suscité un intérêt critique de la part d'Henri Curzon (1902) et de Léon de 
Lanzac de Laborie (1911). Cependant la transcription partielle de ces critiques a transformé l'analyse à laquelle cette réécriture a donné lieu: Patrick Berthier déclare que la réécriture comporte un ajout « dans la scène 3 du premier acte [de] vingt vers justifiant l'établissement de l'Empire, tout en coupant ailleurs les vers qu'il [Esménard] juge indésirables » (p. 259). Dans les faits, on a affaire à une réécriture de la réécriture : le document originel est composé de treize pages folio et propose de nombreux ajouts et changements au texte cornélien, et ces modifications entraînent certaines coupes. Ainsi, la réalité de cette réécriture est beaucoup plus complexe que ce que les analyses qui existent actuellement révèlent et la transcription partielle du document a abouti à des jugements maladroits sur l'état de la tragédie classique à l'époque napoléonienne.

Les « Changemens [sic] proposés pour la Tragédie d'Héraclius [sic] », apparemment de Joseph-Alphonse Esménard (voir Lanzac de Laborie, p. 144), conservés aux Archives nationales à Paris sous la côte F/7/4233, n’ont jamais été étudiés en comparaison avec l'exemplaire originel de la ComédieFrançaise auquel ils font référence (Corneille, s.d.) et qui confirme l'héritage classique réécrit qu'a reçu l'époque napoléonienne. Leur transcription intégrale, en tenant compte des réécritures contenues dans le document lui-même, et même les petits changements, permet un examen des multiples fonctionnements de la réécriture. En nous rappelant les intérêts de l'Etat et du public, notre étude envisagera la réécriture du point de vue de la censure et de la propagande mais la considérera aussi comme une «traduction temporelle » répondant à un nouveau public qui parle une langue révolutionnée. 


\section{La réécriture en tant que censure}

Quoique la réécriture de pièces préexistantes ait été une pratique commune à l'époque, les «Changemens » agissent comme une censure officielle. "La censure " est une notion complexe et multiforme, dotée d'une longue histoire. Sa définition est plutôt problématique, comme le rappelle l'ouvrage de Robert Darnton (p. 17). Pourtant, il s'agit ici de la censure institutionnelle: les archives de la Police attestent un «système général des changemens ordonnés » (Changemens, s.d., p. 6), c'est-à-dire la suppression officielle de certains vers à cause de leur contenu. Par conséquent, l'étude des éléments qui remplacent les «Changemens » est essentielle pour déterminer le fonctionnement de cette réécriture.

La réécriture d'Héraclius crée une image transformée du pouvoir non héréditaire. Dans la version de 1647, la tragédie s'ouvre avec le discours de Phocas, l'Empereur d'Orient, qui raconte qu'il n'est pas celui qui devrait régner mais qu'il a pu monter sur le trône même si le pouvoir héréditaire ne lui revenait pas et y rester en dominant ses sujets par la terreur. Dans le discours réécrit, les vers suivants disparaissent :

Surtout qui, comme moi, d'une obscure naissance Monte par la révolte à la Toute-puissance, Qui de simple soldat à l'Empire élevé (Corneille, 1984, I, 1, v. 911)

de même que "L'Empire était chez nous un bien héréditaire » (1 1, 2, v. 185). La suppression de ces vers n'a rien de surprenant étant donné qu'ils rappelleraient au public que Napoléon, l'Empereur contemporain, n'était pas né en tant que chef d'État et que même ses origines françaises étaient contestables du fait de sa naissance en Corse l'année même où 
elle est passée sous domination française. De plus, «l'Empire élevé » est remplacé par «Sans combats et sans gloire» (Changemens, s.d., p.1) pour éviter que le public puisse assimiler Napoléon à Phocas.

La conception napoléonienne du pouvoir est également mise en avant par les changements dans le discours de Pulchérie. En tant que fille de l'ancien empereur Maurice - le chef d'État que Phocas a dépossédé de son pouvoir -, Pulchérie menace le pouvoir héréditaire que Phocas souhaite transmettre à son fils, Martian. Bien que le pouvoir de Pulchérie soit un grand enjeu dans l'action de la pièce (c'est ce pouvoir qui conduit à son mariage avec Martian et au dénouement de la tragédie), le rôle actif de Pulchérie est sévèrement raccourci. Lors de son entrée à la deuxième scène du premier acte, les changements ôtent à Pulchérie la possibilité de s'exprimer comme une femme indépendante qui pourrait régner. Pulchérie n'est plus la «fille de l'Empereur » comme elle se présentait dans son discours de 1647 (Corneille, 1984, 1, 2, v. 116), ce qui est une première atteinte à son propre droit au trône. De la même manière, le reproche de Pulchérie selon lequel elle serait la proie de Phocas est supprimé, ce que confirme la coupe des trois vers suivants : "Tu ne me rends mon bien, que pour te le donner.» (Corneille, 1984, 1, 2, v. 128) et "Cette feinte douceur, cette ombre d'amitié, | Vint de ta politique, et non de ta pitié. » (1, 2, v. 135-136). Dans la version de 1647, la tirade de Pulchérie en I, 2 souligne le fait que Phocas se sert d'elle pour des raisons politiques et démasque la brutalité d'un usurpateur par l'exploitation d'une victime de toute évidence innocente. Ainsi, ces suppressions métamorphosent la représentation du pouvoir non héréditaire. 
En outre, les changements éliminent le fait que Pulchérie refuse de croire que Phocas est «du bien de [s]es aïeux! » (Corneille, 1984, 1, 2, v. 176) ainsi que cet ordre: "Tyran, descends du Trône, et fais place à ton maître. » (1, 2, v. 234). En réécrivant ces vers, le texte représenté est ainsi dénué de toute allusion qui pourrait inviter les femmes à se révolter contre le pouvoir masculin autoritaire qui avait été restauré avec la domination masculine sur la femme dans le Code Civil de 1804; la réécriture reflète donc les changements sociaux de l'époque. Quoique le sujet de la tragédie traite toujours de l'usurpation du pouvoir et de la rivalité entre les deux familles, ce qui pourrait rappeler la situation de Louis XVIII, la suppression de tels vers, couplée à une nouvelle conception du pouvoir, est plus que commode pour le régime napoléonien. En continuant de réduire les applications au contexte contemporain, elle évite que la représentation ne soit perturbée. Au fil du texte, ce genre d'expression est de moins en moins toléré, puisque, tandis que, dans les premiers discours de Pulchérie, les vers problématiques qui traitent du pouvoir héréditaire et de ses droits en tant que fille d'Empereur sont retravaillés, lorsqu'il s'agit de sa lutte continue contre Phocas, plus tard dans la pièce, les «Changemens » coupent le texte de départ sans y ajouter une nouvelle vision. Ainsi, les « Changemens » font disparaître les vers suivants :

Toi, si quelque remords te donne un juste effroi, Sors du Trône, et te laisse abuser, comme moi,

Prends cette occasion de te faire justice. (Corneille, 1984, 1, 2, v. 251-253)

Cette suppression est d'autant plus remarquable que plusieurs membres du public tenaient les exemplaires de la pièce à la main (voir Lanzac de Laborie, p. 145), tandis que 
d'autres la connaissaient de mémoire. Le fait de couper plutôt que de proposer de nouveaux vers rendait donc évident le caractère intolérable du passage. Or, apparaît ici un autre type de hiérarchie : il ne s'agit plus seulement de la domination des hommes sur les femmes mais bien de celle du chef d'État sur ses sujets. À l'instar des nobles de l'Ancien Régime, Pulchérie a perdu la majorité de ses biens hérités de son sang noble. Pulchérie reste noble mais la réécriture la rend plus assujettie à l'empire contemporain. La réécriture amadoue ainsi les problématiques impériales empruntées tant à Héraclius qu'à l'époque contemporaine.

La hiérarchie des sexes et du pouvoir continue à s'exprimer dans la transformation appliquée à la présentation de Pulchérie. Alors que, dans le texte de 1647, cette fille impériale exprime sa «liberté» et sa capacité à "[se] défendre » (Corneille, 1984, 1, 2, v. 111-112), ces vers sont retravaillés dans les « Changemens » pour exprimer les soins de Phocas et son respect du malheur de Pulchérie (s.d., p. 3). En supprimant sa capacité à prendre soin d'elle-même en tant que femme et en la plaçant sous la tutelle masculine de Phocas, la femme est présentée comme un sujet se pliant aux volontés de l'homme et même, pourrait-on dire, de l'homme d'État et donc du régime. Ainsi la correspondance entre Pulchérie et la société napoléonienne est-elle à nouveau mise en lumière.

Il est important de noter que, dans le texte de départ, Pulchérie avait «contredit» (Corneille, 1984, III, 1, v. 788) le mariage tandis qu'elle l'a « rejeté » dans les « Changemens » (s.d., p. 9). Certes, cette réécriture est infime mais le verbe « contredire » donne davantage de pouvoir à Pulchérie en tant que figure d'opposition tandis que le verbe « rejeter » exprime la 
position supérieure de Phocas, dont la volonté prime sur elle. Quoique les efforts de Pulchérie pour échapper à son mariage avec Martian soient représentés dans le texte réécrit, il y a également la suppression de certains vers sur le passage où elle souligne que ce mariage lui est imposé. La suppression de ce refus rend l'image de Pulchérie plus conforme aux volontés du régime napoléonien exprimées dans le Code Civil. Pourtant, si l'on revient à la deuxième opposition entre chef d'État et sujet, il est clair que la métamorphose de Pulchérie en faveur du régime peut aussi influencer le public lors de la représentation et, par conséquent, la réécriture de son image de fille fougueuse en fille davantage soumise au régime concorde avec le changement de subjectivité et avec le contrôle augmenté sur l'individu que souhaitent imposer le Code Civil et le régime napoléonien. En conséquence, la comparaison des «Changemens» avec les passages réécrits permet de constater quels sentiments étaient intolérables pour le régime napoléonien et les images qu'il voulait transmettre à la nouvelle ère.

\section{La réécriture en tant que propagande}

Ayant établi que la réécriture permettait la suppression des passages problématiques à la censure impériale, il faut ensuite examiner ce qui les a remplacés. La manipulation de l'image de Pulchérie en tant que femme et sujet a déjà révélé comment certains extraits ont été entièrement coupés, une réduction qui est toujours une réécriture. Dans la majorité des cas, le passage éliminé a été remplacé par de nouveaux vers et cette réécriture a tendance à transformer le sens du texte de départ pour en diriger les allusions ainsi que les applications et à étirer la «vérité 
historique » classique, que Corneille avait déjà manipulée luimême, pour renforcer la puissance du régime napoléonien. Robert Holtman soutient que Napoléon est le père de la propagande moderne (voir p. 246), c'est-à-dire qu'il a su orienter suffisamment les esprits pour modeler l'opinion publique à son avantage et à celui de son régime (voir Holtman, p. xi et 244), utilisant entre autres le théâtre pour transformer son pouvoir en spectacle national et international (voir Chaillou, p. 17, 157-174 et 301-316; Triolaire, p. 351-414). D’après les mémoires de l'époque, le théâtre était un des seuls lieux où l'on pouvait voir Napoléon en dehors des parades (voir Blagdon, p. 70), ce qui nourrissait la comparaison entre Napoléon et le comédien vedette de l'époque, François-Joseph Talma, à tel point que, d'après Mara Fazio, Talma est devenu la doublure théâtrale de l’Empereur (p. 117 et 132). De surcroît, Napoléon surveillait le répertoire des pièces représentées et relisait les pièces lui-même. Il conseillait aussi Talma sur sa façon de prononcer les vers.

Dans l'analyse de la réécriture en tant que propagande, l'état matériel du document des « Changemens » devient très important. Lors de la tirade de Pulchérie en I, 2, les changements proposés sont rayés et un becquet avec une réécriture plus longue est collé à côté. L'analyse de cette scène réécrite a déjà permis de constater comment l'esprit argumentatif de Pulchérie est supprimé pour diminuer sa capacité de troubler l'ordre établi. À la place de l'expression de son pouvoir féminin, Pulchérie commence à exposer son héritage brillant :

Mon père appui du trône et vengeur de l'État,

Par Tibère adopté, choisi par le Sénat,

Couronné mille fois des mains de la victoire,

Transmit à ses enfants les titres de sa gloire. (Changemens, s.d., p. 4) 
Pulchérie est ainsi le fruit d'un empereur qui a gagné le pouvoir de manière héréditaire, légale et militaire, une triple assurance qui renforce ses droits au pouvoir qu'il transmettra ensuite à ses enfants. Cette description du père de Pulchérie, représenté à plusieurs reprises comme un grand monarque, fait écho à Napoléon, qui est arrivé au pouvoir par ses conquêtes militaires, a gardé sa place et est devenu Premier Consul, puis plus tard Empereur d'un empire héréditaire, par approbation légale du gouvernement français. Ces quatre premiers vers sont les mêmes dans la première réécriture et dans le becquet. Pourtant, le becquet contient onze vers ajoutés qui ne figurent pas dans le premier changement et qui détaillent les règnes de Trajan, Théodose, « le grand Constantin » soutenu par Dieu lors de ses batailles, un héritage qu'a reçu Maurice. Quoique ces noms soient mentionnés ailleurs dans la tragédie de 1647 en référence aux souverains orientaux, leur image dans la réécriture napoléonienne vise à établir une généalogie en évoquant Trajan, Théodose $1^{\mathrm{er}}$ et Constantin, empereurs romains tous les trois. Au cas où l'application n'était pas suffisamment claire, la nouvelle réécriture cite «le peuple Romain », un adjectif qui n'apparaît que dans les «Changemens ». Malgré les évolutions du décor et du costume, l'époque napoléonienne avait toujours une conception assez floue de "l'antique », qui pouvait être à la fois romain, grec et oriental (voir Lavallée, p.113). Avec l'adjectif «romain» des "Changemens », la tragédie d'Héraclius est désormais resituée dans un lieu imaginaire où l'Orient antique fait partie de l'Empire romain. Cet imaginaire unit le public postrévolutionnaire à cet héritage antique, et Napoléon aux empereurs romains. Cette transformation est d'une très grande importance parce que, tout comme Corneille a manipulé la vérité historique 
pour Héraclius en ce qui concerne la généalogie et la durée des règnes (1984, p. 355-356 et 360-361), le régime napoléonien s'appuie sur la poétique classique et l'espace du théâtre pour engager sa propagande. Cette propagande confirme l'insertion de Napoléon dans une lignée de souverains légendaires, analysée par R. S. Alexander (p. 459). Pour Napoléon, l'héritage que fournissent ces souverains est essentiel afin de renforcer sa position comme Empereur des Français, et d'autant plus dans le cas de la tragédie classique développée à partir des modèles anciens : il lui permet de jouer sur les racines de la France et de l'Empire romain, ce qui réduit les inquiétudes sur son origine corse et rapproche le public parisien du peuple romain cité dans les «Changemens ». Cet ajout du becquet témoigne du pouvoir de se légitimer et de la nécessité de développer sa propagande.

Après avoir renforcé la position de Napoléon par l'exposition de son héritage symbolique et légendaire, la prochaine grande réécriture pour I, 3 est un écho aux discours de la société postrévolutionnaire, qui renforce la position de Napoléon. Cette réécriture, ajoutée aux « Changemens » sur une feuille volante, remplace l'argument d'Héraclius qui se croyait Martian, dont la naissance, en tant que fils de Phocas, devrait assurer sa position sans épouser Pulchérie. Dans la réécriture, Martian (Héraclius) se moque de Phocas, qui croyait qu'il ne pouvait régner que grâce à une femme, un discours qui renforce le pouvoir masculin. Les vers ajoutés font directement référence à la situation de l'époque napoléonienne: "Sans contester ici les droits de ses Ancêtres,| Combien de fois l'empire a-t-il changé de maîtres? » (Changemens, s.d., p. 5, feuille volante, p.1). Cette déclaration reconnaît l'importance du passé dans la construction du présent, ce qui permet au 
régime napoléonien de s'appuyer sur des comparaisons avec la mémoire collective de Louis XIV d'une part et du monde ancien d'autre part; tout en soulignant l'évolution de l'histoire d'un pays, c'est-à-dire les changements de pouvoir, ce qui de nouveau renforce la position de Napoléon. Cette image confirme la transformation du public parisien, des sujets royaux et des citoyens en un public napoléonien. Ce désir de rapprocher le sujet de la tragédie et la situation contemporaine est attesté dans les vers suivants :

Élevant un héros au rang des souverains,

N'a-t-il pas, au déclin d'un siècle de mollesse,

De l'état Languissant Ranimé la Vieillesse? (Changemens, s.d., p. 5 , feuille volante, p. 1)

Ces vers reflètent le discours contemporain, doublement politique et littéraire, sur la décadence du XVIIIe siècle sous Louis XV et Louis XVI, qui a conduit au bouleversement de la Révolution et soulignent comment Napoléon a rendu la gloire à la France. Cet accomplissement est rappelé de nouveau par «Les Dignes Empereurs dont nous suivons la Trace » et par l'introduction de l'espace ambigu des « romains ». Pourtant, ce n'est pas uniquement $d u$ souverain que la réécriture propagandiste traite. Martian (Héraclius) parle des « Enfans de la fortune, Enfans des légions, | [qui] Comptaient, au lieu d'Ayeux, leurs grandes Actions » (Changemens, s.d., p. 5, feuille volante, p. 2). Ici, de même que dans la pensée de Napoléon où la politique a remplacé ce que le destin était autrefois (voir Lecomte, p. 420), c'est le public, la société postrévolutionnaire, qui doit agir dans l'intérêt du gouvernement. Le chef d'État est responsable de sa fonction et il n'en hérite plus automatiquement comme c'était le cas auparavant. Il est signifiant que cette réécriture qui cible la subjectivité du public 
soit ajoutée aux «Changemens » sur une feuille volante, écrite d'une main différente et avec un autre symbole pour "vers ajouté » que celui sur la légende du document. Il s'agit d'une réécriture ajoutée aux "Changemens», ce qui montre le pouvoir que ces changements possèdent et émettent. La salle du théâtre apparaît désormais comme un espace flou où les importantes réécritures du premier acte essaient d'influencer la subjectivité du public parisien pour renforcer la généalogie de l'empire, que ce soit celui d'Héraclius ou celui de la France.

\section{La réécriture d'une réécriture}

Pourtant, ces réécritures ne sont pas les premières, ni les dernières. Il est intéressant de remarquer que, dans l'édition d'Héraclius de Fages parue en 1807 et appartenant à la Comédie-Française, le discours de Martian (Héraclius) de 1, 3 que la réécriture propagandiste sur la feuille volante remplace est effacé, indiquant une certaine continuité dans les changements, même après la chute de Napoléon (Corneille, $1807, \mathrm{I}, 3)$. D'autre part, la première scène du cinquième acte en stances ne paraît pas et celle qui serait la deuxième scène commence ici avec le vers « Eh! quoi? c'est le tyran, c'est lui qui vous envoie, » (Corneille, 1807, p. 38) à l'inverse de «Ô Ciel! Quel bon Démon devers moi vous envoie» dans l'édition de 1647 (Corneille, 1984, V, 2, v. 1555). De la même manière, le texte continue de subir des transformations : les deux premiers discours du troisième acte sont rayés, ce qui correspond à une trentaine de vers (Corneille, 1807, p. 20). 
Ces changements de 1806-1807, manuscrits pour la représentation ou imprimés pour le public, ne sont pas les premiers; ils s'inscrivent dans une tradition déjà existante où un dramaturge peut réécrire ses propres ouvrages à moins que la réécriture ait lieu de manière posthume. La transcription entière des "Changemens » et leur étude comparative avec l'exemplaire de la Comédie-Française auquel ils font référence révèlent que les changements de scène dans l'exemplaire de la Comédie-Française reproduits dans les «Changemens » ne sont pas les mêmes que ceux de l'édition de 1647 ou que ceux figurant aujourd'hui dans les éditions critiques. Par exemple, dans l'édition de la Comédie-Française, II, 4 commence par le vers « Exupère, Madame, est là qui vous demande. » (Corneille, s.d., p. 338), ce qui fait partie de II, 3 dans la version de 1647, qui fournit l'édition critique moderne (Corneille, 1984, II, 3, v. 577). En regardant d'autres exemplaires du théâtre de Corneille de la fin du XVIIIe siècle, il est évident que ce changement de scène décalé est commun (Corneille, 1774, p. 131; Corneille, 1795, p. 277). En effet, le texte d'Héraclius, censé reposer sur les règles classiques (notamment en ce qui concerne les changements de scène), a en réalité déjà subi des modifications, que la représentation et l'impression de la pièce continue à transmettre à de nouvelles générations, la suivante étant celle des romantiques. Ces modifications sont d'autant plus intéressantes qu'elles agissent sur le texte à un moment où ces règles sur la liaison des scènes et les actes sont à la fois remises en cause par le romantisme naissant (Schlegel, 1865, p. 371-372) et réimposées par la critique littéraire, tel le critique Julien Louis Geoffroy (1743-1814), qui réclame un retour au respect de ces règles. Quoique contestées, les règles sont essentielles dans la conception napoléonienne de la 
tragédie dite classique, comme le montrent les vingt-six " conditions » pour une tragédie dans les cours de Népomucène Lemercier (1817, p.177-179). À partir de ces documents, certains éléments compris comme les règles semblent ne pas être de la main de Corneille, ce qui indique les transformations auxquelles les tragédies dites « classiques » ont été assujetties.

\section{Vers une " traduction temporelle»}

Depuis plusieurs siècles, la tragédie française a été associée à la maxime "plaire et instruire ». Quoique le régime napoléonien ait été conscient de ces volontés, il était également vigilant quant aux attentes du public, qui devait s'unir pour reconstruire la nation française après les bouleversements de la Révolution. Curzon et Lanzac de Laborie ont préféré ignorer les petites réécritures malgré leur importance. Certes, l'ampleur de chaque changement peut sembler insignifiante, mais en reformulant le vers ou en changeant un mot pour un autre, ces corrections rendent la tragédie classique et vieillissante de nouveau accessible. Ces réécritures peuvent être considérées comme une "traduction temporelle» dans le sens du latin «traduco», puisqu'elles font passer Héraclius de l'Ancien Régime à la France révolutionnée: l'écart n'est pas géographique mais temporel. Parfois, un vers est reformulé pour faciliter sa compréhension : "Le Peuple en sa personne aime encore, et révère » (Corneille, 1984, I, 1, v. 57) devient «En elle tout le peuple aime encore et révère » (Changemens, s.d., p. 2); "Si pour en voir l'effet, tout me devient contraire» (Corneille, 1984, I, 1, v. 74) se métamorphose en «Quand je veux l'accomplir tout me devient contraire. » (Changemens, s.d., 
p. 3) D'autres fois, le changement peut se limiter à un mot : les vers "Tire chez vous l'amour, qu'on garde pour son nom » (Corneille, 1984, I, 1, v. 72) est réécrit pour commencer par le verbe "porter», qui est plus naturel pour l'époque napoléonienne (Changemens, s.d., p. 3). De même, «ma haine est juste » (Corneille, 1984, I, 2, v. 207) est remplacé par «ma haine est équitable » (Changemens, s.d., p. 5). Cela s'explique par l'évolution des adjectifs « équitable » et «juste» : alors que « équitable » garde un sens juridique, le sens «juste» devient trop diffus (voir Dictionnaire de l'Académie Française, 1762, 1798 et 1835). La réécriture sert donc à traduire un langage ancien au nouveau public de la France révolutionnée.

Il arrive que le changement d'un mot rende le vers plus tragique: «En ce piteux état quel conseil dois-je suivre? » (Corneille, 1984, IV, 3, v. 1363) devient «En ce funeste état» (Changemens, s.d., p.11). Cette petite réécriture est influente grâce à sa modification du sens tragique du vers à un moment où l'on débat des émotions du genre tragique. D’après le cours de Lemercier donné sur la tragédie à l'Athénée de Paris en 1810, bien que la pitié reste au cœur de la tragédie, elle s'est métamorphosée au cours du XVIIIe siècle et on considère qu'elle peut susciter des larmes vulgaires, ce qui n'est pas digne de la tragédie telle qu'elle est définie à ce moment-là (1817, p. 237238). D'ailleurs, toute la première scène du cinquième acte est coupée, une scène composée de stances lyriques et non pas d'alexandrins, qui étaient considérés comme les meilleurs vers tragiques en français (Lemercier, 1817, p. 476). Par conséquent, la réécriture répond aux exigences du public vis-à-vis du genre, surtout lors des décrets sur le théâtre de 1806 et de 1807, qui ont de nouveau formalisé les genres. 
De surcroît, avec la suppression de quelques archaïsmes, la réécriture sert souvent à rendre plus clairs les passages complexes qui traitent de l'action. Avec son jeu sur les fausses identités et son inscription dans un contexte complexe, Héraclius n'est pas la tragédie la plus simple à comprendre. Corneille en était conscient mais maintenait que cette confusion ne nuisait pas à l'action de la pièce (1984, p. 356). Pourtant, les « Changemens » simplifient les passages qui paraîtraient denses au public napoléonien. Par exemple, « Ce Prince » est remplacé par «Martian» afin de préciser de qui il est question. Le discours d'Eudoxe, la fille de Léontine, constitue un autre exemple: le sacrifice du fils de Léonice lors de l'échange de Martian et d'Héraclius, qui est un événement clé de l'intrigue, est mis en lumière par les censeurs, car la version de 1647 «empêche le commun des spectateurs de suivre la marche d'Héraclius» (Changemens, s.d., p. 7). La réécriture tragique facilite la compréhension du texte et le rend plus conforme aux attentes du public.

La société napoléonienne a besoin de ces changements parce que plusieurs de ses membres, à cause des bouleversements de la Révolution, n'ont pas reçu d'éducation classique. La langue française avait d'ailleurs beaucoup changé lors de cette décennie et le sens de certains mots avait été transformé, sans parler de la nouvelle prononciation (voir Kotzebüe, p.231). Or, la société postrévolutionnaire avait toujours besoin de la tragédie de l'ancien régime: la tragédie classique a été diffusée à travers la France révolutionnée par le gouvernement à la suite du décret du $1^{\text {er }}$ mai 1802 dans les lycées et les manuels scolaires mais il ne s'agit pas ici d'un fardeau. En effet, il y avait une vraie demande publique pour la tragédie classique, comme le confirment les nouvelles éditions de 
luxe du théâtre de Corneille et de Racine publiées par Didot en 1801, ou encore la circulation du feuilleton du Journal des débats, devenu plus tard le Journal de L'Empire. Les articles sur le théâtre en général et sur la tragédie classique en particulier de Geoffroy $\mathrm{y}$ atteignaient entre 32000 et 100000 lecteurs (voir Follain, p. 78; Gengembre, p. 80). À partir des analyses de David Bell sur la construction de la nation française lors de la Révolution, il est possible de maintenir qu'après la Révolution, la tragédie classique était partie prenante de l'héritage commun dont la société française bouleversée avait besoin pour la reconstruction de la nouvelle nation française postrévo-lutionnaire (p. 21). Plusieurs publications telles que Des changemens adoptés au Théâtre Français pour la tragédie de Nicomède de P. Corneille, Un changement proposé pour la Tragédie de Polyeucte, du même auteur de François Andrieux (1759-1833) de 1805 et Corneille, Six tragédies retouchées pour le théâtre de 1802 proposent des changements et attestent la peur de la perte et de l'oubli de la tragédie classique. Les changements sont effectués pour que la tragédie reste courante et compréhensible, ce qui conduit à la refondation des canons, encore un élément central pour la culture nationale. En conséquence, il est possible d'envisager les changements d'Héraclius comme une "traduction temporelle» de la langue ancienne à un registre compréhensible pour les locuteurs de l'époque, tout en demeurant classique et historique, pour la nouvelle nation française.

Avec l'analyse de cette réécriture napoléonienne d'Héraclius, il apparaît que le fonctionnement de la réécriture est multiple. D'une part, elle est plutôt officielle dans le sens étatique : elle sert à enlever les vers problématiques qui pourraient nuire au 
régime et offre une occasion de propagande. Même si cette réécriture peut être considérée comme un outil de censure et de propagande destiné à influencer le public, il est clair que les changements répondent aussi aux attentes de ce dernier si bien que cette réécriture peut être comprise comme une «traduction temporelle». À partir des arguments de Lefevere selon lesquels toute réécriture est idéologique dans son rapport $\mathrm{au}$ social et au politique, il est possible de constater qu'en facilitant la compréhension d'une partie de la culture commune, cette réécriture aide à consolider les rapports entre le public et le gouvernement pour contribuer à la reconstruction de la nation française conçue par Bell. Toute la réécriture vise à influencer la réception de la pièce par le public, à tel point que les notions de «censure » et de " propagande » commencent à se confondre dans leur volonté de transformer la subjectivité du public. Certes, Héraclius n'est qu'un exemple de la réécriture de la tragédie dite "classique », mais il manifeste des enjeux complexes à l'époque et le rôle de la culture à l'ère napoléonienne. En ce qui concerne l'histoire du théâtre, cette réécriture montre que les Français avaient bel et bien appris comment modifier leur théâtre avant l'arrivée de Shakespeare avec les tournées des années 1820 et l'avènement du drame romantique (voir Naugrette, p.62-63). À une époque où « classique » et « romantique » s'affrontent et se (re)définissent, la tragédie dite « classique » est réécrite et ajustée aux attentes $\mathrm{du}$ public contemporain, remettant ainsi en cause l'essence même du « classicisme ».

Il reste à donner la transcription entière des «Changemens proposés pour la Tragédie d’Héraclius », qui révéleront la véritable étendue de la réécriture officielle de la tragédie « classique » représentée. 
La retranscription correspondant à cet article se trouve sur le Warwick Research Archive Portal. Pour la consulter, veuillez suivre ce lien : http://wrap.warwick.ac.uk/78191/ (WRAP_Analyses_CSiviter_Héracius_transcription.pdf).

\section{Bibliographie}

(1806), « Changemens proposés pour la tragédie d'Héraclius », Paris, Archives nationales, F/7/4233.

(1804), Code Civil des Français, édition seule et originale, Paris, l'imprimerie de la République.

(1762), Dictionnaire de l'Académie Française, <http://artflsrv02.uchicago.edu/cgi-

bin/dicos/pubdico1look.pl?strippedhw=èquitable\&headwor

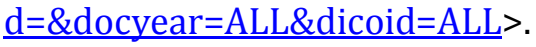

(1798), Dictionnaire de l'Académie Française, <http://artflsrv02.uchicago.edu/cgi-

bin/dicos/pubdico1look.pl?strippedhw=èquitable\&headwor

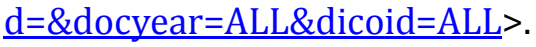

(1835) Dictionnaire de l'Académie Française, $<$ http://artflsrv02.uchicago.edu/cgi-

bin/dicos/pubdico1look.pl?strippedhw=èquitable\&headwor $\underline{\mathrm{d}=\& \text { docyear=ALL\&dicoid=ALL. }}$.

AleXANDER, R. S. (2000), «The hero as Houdini: Napoleon and $19^{\text {th }}$ century bonapartism $"$, Modern \& Contemporary France, vol. 8, no 4, p. 457-467

ANDRIEUX, François (1805), Anaximandre, ou le Sacrifice aux Grâces, comédie en un acte en vers de dix syllabes, Nouvelle édition, corrigée par l'auteur. On y a joint: 1 des changements adoptés au Théâtre Français pour la tragédie de "Nicomède", de P. Corneille; 2 un changement proposé pour la tragédie de "Polveucte", du même auteur, Paris, Colin. 
BELL, David A. (2001), The Cult of the nation in France, inventing nationalism, 1680-1800, Cambridge, Harvard University Press.

BERTHIER, Patrick. (2014), Le Théâtre en France de 1791 à 1828. Le sourd et la muette, Paris, Honoré Champion, coll. «Dictionnaires et références ».

BLAGDON, Francis. (1803), Paris as it was and as it is; or a sketch of the French capital illustrative of the effects of the revolution, t. 1 , Londres, Baldwin.

Chaillou, David. (2004), Napoléon et l'opéra. La politique sur la scène 1810-1815, Paris, Fayard.

Chaouche, Sabine. (2013), La Mise en scène du répertoire à la Comédie-Française: 1680-1815, 2 vol., Paris, Honoré Champion, coll. « Dix-huitièmes siècles ».

CoRneille, Pierre. (s.d. [1647]), Héraclius, dans Chefs d'œuvres de Corneille, vol. 2, s.l. [BMCF 1. HER Cor «Extr. De Chef d'œuvre de Corneille, t. II, p.305-398, seconde moitié du XVIII' s. »].

—. (1774), Théâtre, t. IV, Genève, s.n.

—. (1795), Théâtre de P. Corneille, t. VI, Paris, Didot l'aîné.

- (1801), CEuvres de P. Corneille, avec le commentaire de Voltaire sur les pièces de théâtre, et des observations critiques sur ce commentaire, par le citoyen Palissot, Paris, Didot l'aîné.

-. (1802), Six tragédies [et Héraclius, 7e tragédie...] de Pierre Corneille retouchées pour le théâtre. Edition plus correcte, Paris, [n.pub].

—. (1807 [1647]), Héraclius, tragédie en cinq actes et en vers, Paris, Fages.

- (1984), CEuvres complètes, textes établis, présentés et annotés par Georges Couton, vol. 2, Paris, Gallimard, coll. «Bibliothèque de la Pléiade». 
-. (2006), Théâtre, présentation par Christine Noille-Clauzade, vol. 3, Paris, GF Flammarion.

Couton, Georges. (1980) 'Notice sur Le Cid' dans Pierre Corneille, OEuvres complètes, textes établis, présentés et annotés par Georges Couton, vol. 1, Paris, Gallimard, coll. "Bibliothèque de la Pléiade », p. 1449-1480.

CuRzon, Henri de. (1902), « Comment on retouchait Corneille pour le rendre digne de Napoléon, Héraclius à la cour », Bulletin de la société de l'histoire du théâtre, vol. 1, no 3-4, p. 113-121.

DARNTON, Robert. (2014), Censors at work: how states shaped literature, Londres, British Library.

EigenmanN, Eric. (2004), «Cinna sans clémence? La tragédie de Corneille "remise au théâtre" par Voltaire et Tronchin », DixSeptième siècle, vol. 4, no 225, p. 747-755.

FAZIO, Mara. (2011), François-Joseph Talma, le théâtre et l'histoire de la révolution à la restauration, Paris, CNRS.

Follain, Jean. (1969), «Le Journal des débats et ses démêlés avec l'Empire », Europe, no 480-481, p. 77-79.

FRANTZ, Pierre. (2010), " Les dénouements en action au XVIII siècle », dans Mara Fazio et Pierre Frantz (dir.), La Fabrique $d u$ théâtre avant la mise en scène (1650-1880), Paris, Desjonquères, p. 332-342.

GEnetTE, Gérard. (1992 [1982]), Palimpsestes, la littérature au second degré, Paris, Seuil, coll. «Points Essais ».

GENGEMBRE, Gérard. (1999), Le Théâtre français au 19e siècle, Paris, Armand Colin.

Holtman, Robert. (1950), Napoleonic Propaganda, Baton Rouge, Louisiana State University Press.

KotZeBüE, August von. (1805), Souvenirs de Paris en 1804, traduits de l'allemand, sur la deuxième édition; avec des notes, vol. 2, Paris, Barba. 
LANZAC DE LABORIE, L. D. (1911), Paris sous Napoléon. Le Théâtre-Français, Paris, Plon-Nourrit.

LAVALLÉE, Joseph. (1803), Lettres d'un mameluck ou tableau moral et critique de quelques parties de mœurs de Paris, Paris, Capelle.

LECOMTE, Louis-Henry. (1912), Napoléon et le monde dramatique: étude nouvelle d'après des documents inédits, Paris, Daragon.

LEFEVERE, André. (1992), Translation, rewriting, and the manipulation of the literary frame, London, Routledge, coll. «Translation Studies ».

LEMERCIER, Népomucène-Louis. (1817), Cours analytique de littérature générale, tel qu'il a été professé à l'Athénée de Paris, vol. 1, Paris, Nepveu.

MARCHAND, Sophie. (2010), « La mise en scène est-elle nécessaire? L'éclairage anecdotique (XVIII ${ }^{\mathrm{e}}$ siècle) ", dans Mara Fazio et Pierre Frantz (dir.), La Fabrique du théâtre avant la mise en scène (1650-1880), Paris, Desjonquères, p. 40-50.

NAUGRETTE, Florence. (2001), Le Théâtre romantique. Histoire, écriture, mise en scène, Paris, Seuil, coll. « Points Essais ».

RACINE, Jean. (1801), CEuvres de Jean Racine, Paris, Didot l'aîné.

SCHLEGEL, August Wilhelm von. (1865 [1809-1811]) Cours de Littérature dramatique, traduit de l'allemand par Mme Necker de Saussure, Nouvelle édition revue et annotée, 2 tomes, Paris, Lacroix, Verboeckhoven et Cie.

SERGI, Antionio. (1974) "Phèdre corrigée sous la Révolution", Dix-Huitième siècle, vol. 6, p. 153-165.

TRIOLAIRE, Cyril. (2012), Le Théâtre en province pendant le Consulat et l'Empire, Clermont-Ferrand, Presses universitaires Blaise Pascal, coll. «Études sur le Massif central ».

VolTAiRE. (an II/1793-4 [1735]), La Mort de César, tragédie en trois actes de Voltaire, avec les changemens faits par le 
Citoyen Gohier, Ministre de la Justice, représenté au Théâtre de la République à Paris, Paris, L. Cutty.

ZÉKIAN, Stéphane. (2012), L'Invention des classiques, le « siècle de Louis XIV » existe-t-il?, Paris, CNRS éditions.

\section{Résumé}

La réécriture d'Héraclius (1647) de Pierre Corneille en 18061807 constitue un bel exemple de la malléabilité de la tragédie classique au fil du temps, surtout lorsque son statut a été remis en cause par le romantisme naissant. À partir des «Changements ordonnés» de la Police et l'exemplaire de la Comédie-Française, cet article examine les enjeux pour l'État et le public dans la réécriture. Il considère ainsi le rôle de ces changements comme une forme de censure et de propagande, mais aussi comme une traduction temporelle pour l'aboutissement à une culture commune, permettant la reconstruction nationale après la Révolution

\section{Abstract}

The rewriting of Pierre Corneille's Héraclius (1647) in 18061807 is a prime example of 'neo-classical' tragedy's malleability over time, especially as its status was being questioned by the rise of Romanticism. Focusing on the Police's 'Changements ordonnés' for Héraclius and their corresponding copy in the archives of the Comédie-Française, this article examines the stakes for both the government and the public in rewriting classical tragedy. Consequently, it will envisage these changes for their role in censorship and propaganda, but also as a temporal translation for the increased shared culture, allowing for national reconstruction after the French Revolution. 